\title{
Using Citizen Science Gamification in Agriculture Collaborative Knowledge Production
}

\author{
Jonathan Martin \\ LIFIA, Facultad de Informatica, UNLP \\ La Plata, Argentina \\ jonathan.martin@lifia.info.unlp.edu.
}

ar

\author{
Diego Torres \\ LIFIA, Facultad de Informatica, UNLP \\ CIC, Prov. Bs. As. \\ Depto. Ciencia y Tecnologia, UNQ \\ La Plata, Argentina \\ diego.torres@lifia.info.unlp.edu.ar
}

\author{
Alejandro Fernandez \\ LIFIA, Facultad de Informatica, UNLP \\ CIC, Prov. Bs. As. \\ La Plata, Argentina \\ alejandro.fernandez@lifia.info.unlp. \\ edu.ar
}

\author{
Santiago Pravisani \\ LIFIA, Facultad de Informatica, UNLP \\ La Plata, Argentina \\ santiagopravisani@gmail.com
}

Guillaume Briend

Bretagne Développement Innovation

France

g.briend@bdi.fr

\begin{abstract}
ANONYMOUS PROJECT is an international project run by 16 research institutions. The project's main goal is empowering agriculture production systems through innovative knowledge-based ICT solutions to deal with high risk and uncertain conditions. In this context, a Semantic Mediawiki is desired to support the various stakeholders in the agriculture value chain in the task of capturing and sharing agricultural best practices. Applying gamification to Wikis is one strategy to encourage the community to participate, and It could be achieved by attaching Metagame, a gamification layer system. However, It could be risky because the tunning of a gamification system in the production face of the wiki could be perceived by the users as a bug in the system. To avoid this situation, a simulation based on the real historical data of the Agriculture community in Wikipedia from 2001 to 2018 was performed. The simulation revealed that is not obvious to generate a system of awards on the type of actions carried out by the Wikipedia editors. The construction of knowledge is done in very small portions, and some of them can be confused with improvements or corrections instead of contributions. Indeed, It shows the importance of tuning the reward parameters, because that allows advancing in the Metagame rank evolution.
\end{abstract}

\section{CCS CONCEPTS}

- Human-centered computing $\rightarrow$ Wikis; Collaborative and social computing design and evaluation methods; • Theory of computation $\rightarrow$ Exact and approximate computation of equilibria; - Applied computing $\rightarrow$ Agriculture;

\section{KEYWORDS}

Agriculture, Gamification, Collaborative Knowledge Building

ACM acknowledges that this contribution was authored or co-authored by an employee contractor or affiliate of a national government. As such, the Government retains a nonexclusive, royalty-free right to publish or reproduce this article, or to allow others to do so, for Government purposes only.

Interacción 2018, , Palma, Spain

(C) 2018 Association for Computing Machinery.

ACM ISBN 978-1-4503-6491-1/18/09...\$

https://doi.org/10.1145/3233824.3233859
ACM Reference Format:

Jonathan Martin, Diego Torres, Alejandro Fernandez, Santiago Pravisani, and Guillaume Briend. 2018. Using Citizen Science Gamification in Agriculture Collaborative Knowledge Production. In Interacción 2018: XIX International Conference on Human Computer Interaction, September 12-14, 2018, Palma, Spain. ACM, New York, NY, USA, Article , 8 pages. https: //doi.org/10.1145/3233824.3233859

\section{INTRODUCTION}

ANONYMOUS PROJECT is an international project run by 16 research institutions. Participants represent both academic and nonacademic organizations. The project's main goal is empowering agriculture production systems through innovative knowledgebased ICT solutions to deal with high risk and uncertain conditions.

Communities of practice that shares knowledge in a wiki-way [19] are a promising strategy to deal with risk and uncertainty. In the context of ANONYMOUS PROJECT , a Semantic Mediawiki [9] will be deployed. Its purpose is to support the various stakeholders in the agriculture value chain in the task of capturing and sharing agricultural best practices.

It is well known that a wiki is as good as the community that powers it $[11,19]$. Gamification $[2,4,6]$ is among the strategies being explored to build and sustain a rich and active community of editors $[3,4]$. The wiki will be enriched with a game that rewards editing, engaging in discussion with others, and disseminating. Instead of designing a gamification strategy from scratch, an existing pluggable gamification service will be attached. Metagame $[15,16]$ is a citizen science metagame that can be attached to any crowdsourcing project via an event bus. It adds a layer of gamification that interprets actions in the project as moves in a game.

Attaching Metagame to a project involves: a) identifying events in the project that will act as moves in the game, b) fine-tune the transformation from project events to game moves in order to create an enjoyable experience. These two tasks, although simple in technical terms, require iteration and experimentation. For example, if too many page-edit events are needed in order to get a certain badge the game dynamics will be affected and the resulting experience will not be satisfactory.

Doing the fine-tuning of the game configuration in production (with the real wiki and the real community) is risky. Users could 
perceive changes in the game mechanics as bugs of the system or as unfair changes. In order to launch the production face of the wiki with a stable version of the game requires considering other tunning alternatives based on simulations[8]. We discarded synthetic simulation of users behavior because there were too many variables involved. As an alternative, we adapted the Orta \& Ruiz conceptual framework [13] and then we explored running the game on historical data of an existing wiki, with a community that resembles the one we pretend to generate. In the following sections, we describe such wiki and its community, the adaptation and simulation process, and the observed results.

\section{WIKIPEDIA AND ITS AGRICULTURE COMMUNITY}

Wikipedia is an open multilingual encyclopedia that is built by regular people on the Web. In Wikipedia, any reader can freely edit any of the articles that appear in the encyclopedia, generally without restrictions. Wikipedia is a type of websites called Wiki [11] which allows to a group of people to contribute with small pieces of knowledge to build collective knowledge. In Wikipedia, any person is ready to participate in the edition by adding a piece of text, a link, edit or comment content. The information of Wikipedia is mainly organized in Articles and Categories. An article is a page that has encyclopedic information on it. That means that in Wikipedia only relevant information is contained in it and it does not include another kind of information like dictionary definitions. The quality of Wikipedia articles varies in a range from low-quality candidates for speedy deletion of high quality featured articles. The quality value is determined by elements like the writing style, external academic references, and structure of the information. On the other hand, a Category is a group of articles that share the category title topic.

The activities that a user as an editor can perform are wide. All of them are related to improving the content of an article. For that, Wikipedia offers two editing environments with the same possibilities. Both environments allow editing the content of an article through adding, deleting or modifying text with several formats such as links, sections, bullets, external links, images, tables, etc.

The collaborative knowledge building process that happens in Wikipedia also involves the presence of discrepancies in the edition. For that, Wikipedia includes for each article a discussion section called Talk. In a Talk page of an article, each user can comment on the article content using paragraphs.

Wikipedia logs all the editions of an article in its revision history, a page that contains the change logs from the article creation until the last edition. This feature is useful to roll back when appears a vandalism edition or just to fix an edition mistake. Traversing the revision history of a Wikipedia article allows to recreating all the activity that editors did in the history the article.

\subsection{Agriculture community in Wikipedia}

This article analyses the knowledge building the community of Agriculture category ${ }^{1}$ in the English chapter of Wikipedia ${ }^{2}$. The

\footnotetext{
${ }^{1}$ https://en.wikipedia.org/wiki/Category:Agriculture Accessed on April 25th, 2018

${ }^{2} \mathrm{http}: / /$ en.wikipedia.org Accessed on April 25th, 2018
}

Table 1: Agriculture by type in Wikipedia

\begin{tabular}{lrr}
\hline Category name & \#Sub-categories & \#Pages \\
\hline Aquaculture & 7 & 107 \\
Beekeeping & 11 & 94 \\
Cannabis cultivation & 3 & 22 \\
Commercial farming & 2 & 5 \\
Dairy farming & 6 & 39 \\
Domesticated animals & 18 & 83 \\
Forestry & 30 & 52 \\
Hemp agriculture & 2 & 1 \\
Livestock & 25 & 158 \\
Orchards & 1 & 12 \\
Organic farming & 10 & 76 \\
Permaculture & 4 & 30 \\
Pig farming & 2 & 15 \\
Poultry farming & 3 & 44 \\
Sustainable agriculture & 16 & 184 \\
Viticulture & 4 & 59 \\
\hline
\end{tabular}

whole Wikipedia community includes sub-communities according to their knowledge areas, most of them are identified by the use of categories[17, 18].

The Agriculture category is defined, regarding Wikipedia, as "the process of producing food, feed, fiber an other desired products by cultivation of certain plants and the raising of domesticated animals". It includes 34 sub-categories and 2,394,712 articles that belongs directly or indirectly ${ }^{3}$.

For the sake of this analysis, this article selected the Agriculture by type sub-category as representative of a smaller community of agriculture editors related to the manner of agriculture is produced. This sub-category includes 16 sub-categories. Table 1 details in the first column the name of the category (sub-category of Agriculture by type, the second column the number subcategories and, the last column the number of pages it includes.

\section{METAGAME}

Metagame is a transversal game based on badges rewards with impact in different projects. In these projects, regular people (called players by Metagame) participate performing specific tasks that will be recorded in Metagame and, depending on the kind of action; players receive badges as a reward. Participation in Metagame is indirect in an non-intrusive mode. Player has to participate in a specific project covered by Metagame.

The diversity of badges that could be obtained is directly related to the number of projects subscribed to Metagame. As the main actions and purposes are diverse among Metagame projects, each project subscribed to Metagame has to define their Unit of Work badges that will be awarded by Metagame. For example, in an image classification project the Unit of Work badge could be obtained by a participant after s/he classifies five images; in a map localization

\footnotetext{
${ }^{3}$ Based on PetScan pages analysis with the following configuration https://petscan.wmflabs.org/?language $=$ en $\&$ project $=$ wikipedia $\&$ depth $=10 \&$ categories $=$ Agriculture $\& \mathrm{~ns} \% 5 \mathrm{~B} 0 \% 5 \mathrm{D}=1$ \&interface_language $=$ en \&active_tab $=$ Accessed on April 25th, 2018
} 
Table 2: Levels of expertise in Metagame (Player Rank)

\begin{tabular}{ll}
\hline Player Grade & Necessary badges \\
\hline Visitor & Nothing \\
Explorer & I was here / Welcome back \\
Citizen Scientist (CS) & $\begin{array}{l}\text { Unit of work / Back and forth / } \\
\text { Shout out loud }\end{array}$ \\
Prolific CS & 10 badges \\
- Specialist Prolific CS & x of 10 badges same project \\
- Generalist Prolific CS & y of 10 badges different projects \\
- Net-worker Prolific CS & j of 10 reinforcement badges \\
- Disseminator Prolific CS & k of 10 dissemination badges \\
Committed Citizen Scientist & 1 badge each month \\
Visionary Citizen Scientist & Design a CS game \\
\hline
\end{tabular}

project, the badge could be obtained after a participant marks an issue in the map.

The former example illustrates different levels of effort to achieve a task inspired in a typical citizen science project: classify a picture in the laptop screen requires less effort than walking to detect a problem in the street. The difficulty and magnitude to obtain a badge in each case have directly impacts in Metagame.

Metagame differentiates among three types of action: Contribution, Reinforcement, and Dissemination.

Contribution: These are the main action a player perform in the different projects covered by Metagame. These actions reflect the main goal of the project. For example, in a picture classification project, the action of classifying a picture will be a contribution.

Reinforcement: These are secondary actions or reinforcement actions a player can perform. Represents an opinion or critic of a previous action like a contribution. For example, a player can agree or disagree on a classification criterion.

Dissemination: These are social actions of propagation and diffusion of other activities. Sharing a result on Facebook or Twitter is an example of dissemination.

\subsection{Players Rank}

Each time a player receives a badge, s/he is evolving to a new game level. Players in Metagame are traversing in a narrative about a world of citizen scientist. In the beginning, players initiate as visitor, then as s/he fulfill the objectives set by Metagame, s/he will reach better ranks as "Citizen Scientist" or "Visionary Citizen Scientist". Table 2 shows the complete list of ranks in Metagame. The first column contains the player grade and the second column details the type and amount of badges are necessary to obtain the rank.

The design of ranks in Metagame is not linear, but adopts a curve line form. Level design is based on a narrative where each rank has a name that represents a moment in the life of a scientist. The ranks used on Metagame are:

- Visitor: It is the first rank, through which all players will cross when they start in the Metagame. To move to the next rank, the player has to obtain the first two badges ("I was Here" and "Welcome Back").

- Explorer: Metagame expects that players in this rank learn the mechanics of the game, therefore, to move to the next
Table 3: Wikipedia actions taking into account Metagame

\begin{tabular}{ccl}
\hline Contribution & Reinforcement & Dissemination \\
\hline Add new paragraph & Add talk entry & Share in WP \\
Create new article & Modify paragraph & Add internal link \\
Add reference & Delete phrase or less & Add external link \\
Add headings & Do a minor change & Add redirections \\
Add image & Move phrases & \\
Add table or row & & \\
Add list or elements & & \\
\hline
\end{tabular}

rank the player is required to obtain a badge of each main type: Contribution, Reinforcement, and Dissemination. Once these badges are obtained, the player will go to the next rank.

- Citizen Scientist: The player stops being a rookie, and happens to become a regular player on the platform. The Citizen Scientist is free to obtain any insignia, and to build a habit, in Metagame they are challenged to obtain ten new badges, to advance to the next rank, "Prolific Citizen Scientist".

- Prolific Citizen Scientist: These players are regular in Metagame. They can be specialized according to the type of actions they perform.

- Generalist: Are the Prolific Citizen Scientist who have to receive badges from more than one project in Metagame. This rank motivates players to participate in several projects.

- Specialist: Motivate to obtain a majority of insignias of type Contribution.

- Disseminator: This player profile will be motivated to make diffusion actions.

- Networker:Is the profile of player that generates networking. They obtain a majority of Reinforcement badges.

- Committed Citizen Scientist: This is the maximum level a player could reach. These are faithful players in Metagame. However, this is the only rank where the player must remain active, or otherwise, her o his rank will be revoked and will return to Prolific Citizen Scientist.

\section{PROBLEM DEFINITION}

Attaching Metagame to Wikipedia Agriculture knowledge building project involves several steps. Regarding Conceptual Framework for ITSM Process Improvement [13] the problem of attaching Metagame to the Wikipedia Agriculture knowledge building project could be defined concerning three key activities: define the organization's process, build process simulation model and run the simulation model experimentation. In the following, a detail in the agriculture context of each key term is introduced.

(1) Define the organization's process: identifying actions in the edition of Agriculture pages project that will act as moves in the game such as editions, adding a link, add a heading, delete a paragraph, etc.

(2) Build process simulation model: fine-tune the transformation from project events to game moves to create an enjoyable experience. For this, we have defined two mapping rules: Wikipedia actions to Metagame actions and, level of difficulty for the acquisition of badges. Firstly, the mapping 
Table 4: Points to earn badges in Metagame

\begin{tabular}{cccl}
\hline \# Actions & Action Type & Badge & Badge level \\
\hline 1 & Login & I was here & 1 \\
2 & Login & Welcome back & 2 \\
$2 *$ level & Contribution & Unit of Work & $1 . .10$ \\
$10^{*}$ level & Reinforcement & Back and forth & $1 . .10$ \\
$1^{*}$ level & Dissemination & Shout out loud & $1 . .10$ \\
\hline
\end{tabular}

between the actions in Wikipedia and the actions defined in Metagame. This mapping is detailed in Table 3 were each column represents a list of actions, the first column enumerates the actions that are considered as contributions, the second those considered as reinforcements and the third those as dissemination. Clearly, the speculation in the mapping contemplates adding elements as a clear contribution and the edition of existing things as reinforcements. The dissemination actions are only those that are expressed in the context of Wikipedia, such as the article appears linked in other page or Wikidata project. Unfortunately, disseminations in social networks like Facebook are registered neither in the activity history or other projects of the Wikimedia family. Finally, the levels of difficulty for the acquisition of badges are defined in Table 4 . The first column contains the number of required actions, the second the action type, and then the third column has the badge name and the last column the number of levels for a badge. The column shows how many actions of a type a player have to perform to obtain a badge. For example, the first line must be read as "the player that perform 1 Login will receive the I was here badge with level 1", consequently the third line could be read "the player that performs $4\left(2^{*} 2\right)$ Contributions will receive the Unit of Work badge with level 2". As we can see, we have defined 10 levels for the badges Unit of Work, Back and forth and Shout out loud. The value that multiplies the number of actions in the first column indicates the ease associated with that action. The bigger the multiplier, the easier the action is considered. Finally, as Metagame is defined as a game, Wikipedia "editors" are mapped to Metagame players. In the following of this article, we will use the word "players" to refer Wikipedia editors.

(3) Simulation model experimentation: Running the simulation in Metagame and analysis the badges and ranks obtained by the players.

The following sections detail the methodology process to perform the simulation framework activities.

\section{METHOD}

The simulation in the use of Metagame with Wikipedia Agriculture projects was organized in three tasks. The first task is in charge of obtaining from Wikipedia all the Agriculture articles with its revision history, discussion pages, and other articles that with a link to them. This task takes advantage of the existing information generated by the editors in Wikipedia without need people to create new editions. The next task generates a chronological list of all the

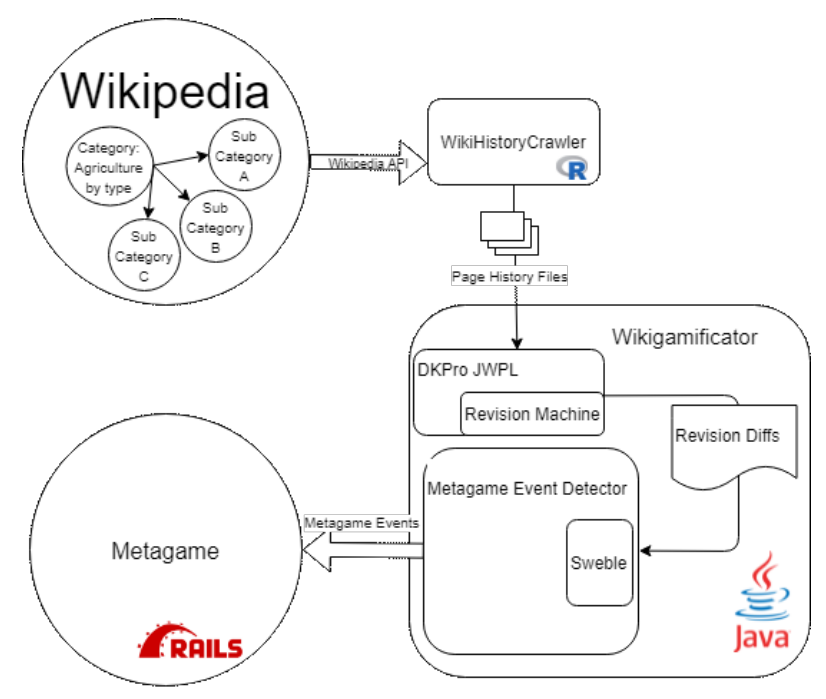

Figure 1: Wikigamification workflow.

edition actions in Wikipedia but regarding Metagame following the rules described in Table 3. This list will be used as input to run the Metagame simulation. Finally, Metagame is configured with the badges acquisition rules as are detailed in Table 4. Figure 1 shows the method and the below sub-sections describe these three tasks.

\subsection{Wikipedia Extraction}

This task obtains from Wikipedia all the revision history of the relevant pages. In this context, pages that directly belongs to the subcategories of "Agriculture by type" (Table 1. Additionally, their talk pages and any Wikipedia page with a link to them are also included. For example, Humus ${ }^{4}$ is a relevant page because it belongs to the category Organic farming, and Organic food is also included because it has a link to Humus article even if Organic food does not belong to a Agriculture by type sub-category. However, as in Wikipedia, an article could belong to more than one category, only articles with disjoint categories were selected.

In order to locally process the relevant pages, we have developed an R script which interacts with the Wikipedia $\mathrm{API}^{5}$ to download the data from the Web. It can be seen in Figure 1 as WikipediaHistoryCrawler.

\subsection{Activities generation}

From the revision history obtained in the former task, the Wikigamificator process generates the chronological list with all the edition actions in Wikipedia but regarding Metagame ${ }^{6}$. First, it detects the changes between revisions to know how much changes an editor made using DKPro JWPL libraries [7]. Finally, the changes were parsed with Sweble[5] and expressed regarding the action listed in Table 3 (for example Add title or move paragraph). We called this list Event data-set. This process is shown in Figure 1 as Metagame Event Detector.

\footnotetext{
${ }^{4}$ https://en.wikipedia.org/wiki/Humus Accessed on April 25th, 2018

${ }^{5}$ https://www.mediawiki.org/wiki/API:Main_page

${ }^{6}$ Not-register editors are grouped with the name "Anonymous"
} 


\subsection{Metagame setup}

The Metagame setup was done as Listing 1. We have considered each sub-category from Table 1 as a specific project. Thus, an editor who edits a page in a specific sub-category is participating in the sub-category project. All of these projects are created in Metagame with the instructions from line 2 up to 22 .

Then, we have defined 10 levels of Contribution, Reinforcement and Dissemination badges and 2 levels of login badges for each project. Lines 25 to 44 in Listing 1.

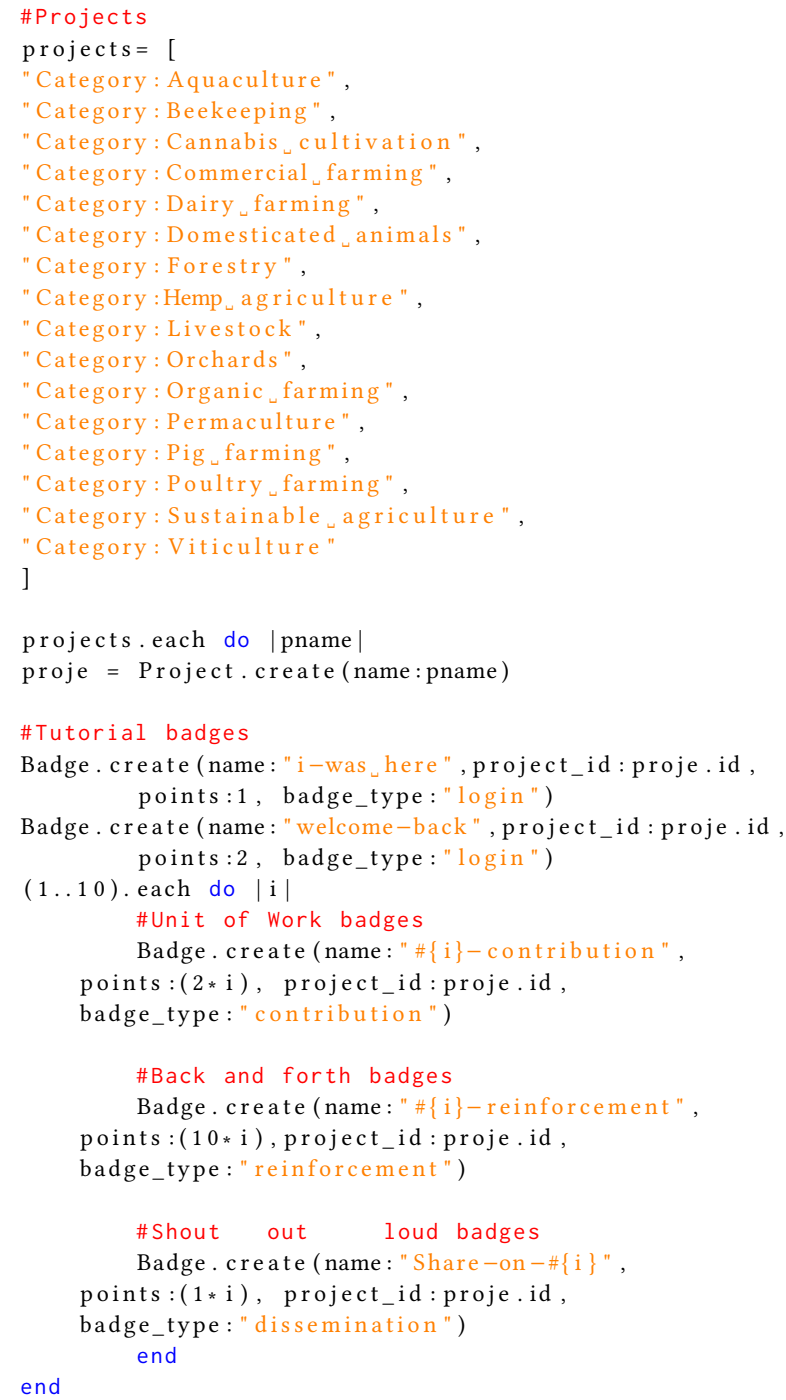

Listing 1: Metagame code setup

\section{EVALUATION}

The evaluation was conducted over the revision history of 4,690 articles including discussions and related articles extracted on April 2018. The Event data-set included 9,939,296 Metagame actions with Anonymous players (8,826,965 without Anonymous). 29,783 players were identified. Those revisions were made between April of 2001 and April 2018, that means revisions of 17 years. All the code and the Event data-set used in the evaluation could be found at https: //github.com/cientopolis/wikigamification.

Anonymous users were avoided in the Metagame simulation. However we have made a previous analysis of Wikipedia activities taking into account Anonymous users.

We have run the evaluation to answer following questions:

- Q1: How was the distribution of the users based on their activity?

- Q2: How much of the events were produced by each kind of users?

- Q3: How was the distribution of the events in the projects?

- Q4: How is the actions evolution along the time?

- Q5: What was the rank distribution of the players in Metagame?

- Q6: How was the delivery of badges over the time?

As mentioned below, the Table 3 was considered for the classification of the Metagame actions. As Metagame requires actions for "login", each time an editor stars a revision was considered a login action.

\section{RESULTS}

From the analysis of the players in the Event data-set, a division in groups of player per the intensity of activity was defined inspired by [10]. The mean, median and standard derivation of the number of actions of each Player were used to selecting the values to split the groups in the following way:

- Tourist is the player that had less than 10 activities, that is less than the median.

- Interested is the player that had more than 10 activities but less than 296 activities, that is less than the mean.

- Average is the player that had more than 10 activities but less than 4924 activities, that is less than the standard derivation.

- Active is the player that had more than 4924 activities.

To answer question Q1, we analyzed the percentage of the total of player that belongs to each group as is shown in Figure 2. In this analysis, Anonymous actions were considered made by only one player. As it is expected, most of the player are tourist (52\%) or interested (39.5\%), and they had done less than 10 actions or 296 actions respectively. On the other hand, the active players are less than $1 \%$.

Continuing with the study of the players to answer the question Q2, Figure 3 shows the percentage of actions that belong to each group of players, in this case taking into consideration the Anonymous player as well. It shows that the majority of actions was produced by the active players $(52 \%)$. In contrast, the tourist players produced only $0.74 \%$ of the actions. Also, the $11.2 \%$ of activities were produced by the Anonymous players. As a conclusion, both Figures 2 and 3 showed that the players with less activity produced more content than the other players. However, it is important to remark the relevance of Anonymous production.

To answers question Q3 we analyze the events distribution per project. Figure 4 shows a bar graph with the analysis. The axis "Categories" details the name of each agriculture type category including the number of articles that it contains and, in the axis "Percentage of Actions" appears a bar with the actions proportions and types. According to the graph, in general, all the categories have 


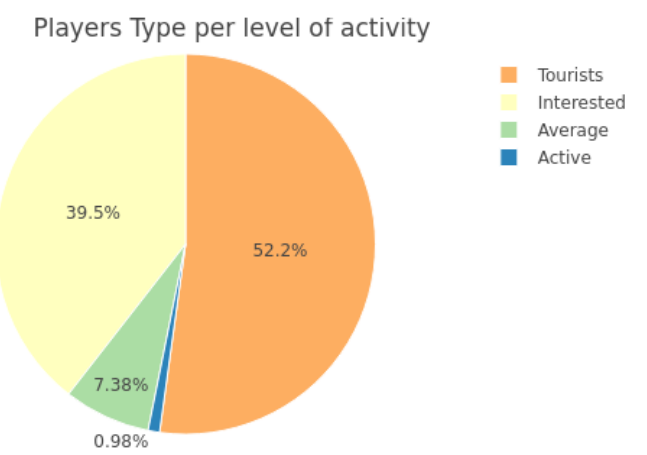

Figure 2: Players type per level of activity.

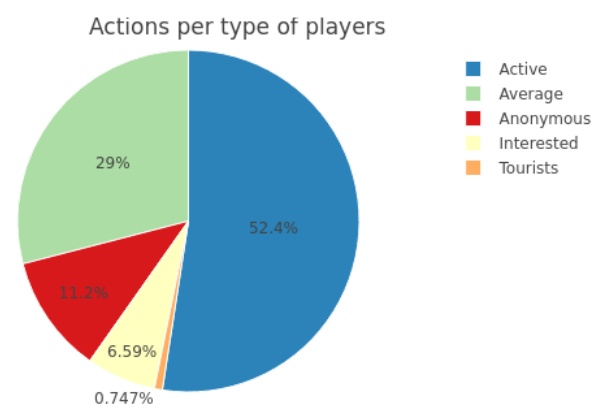

Figure 3: Number of Activities per type of player.

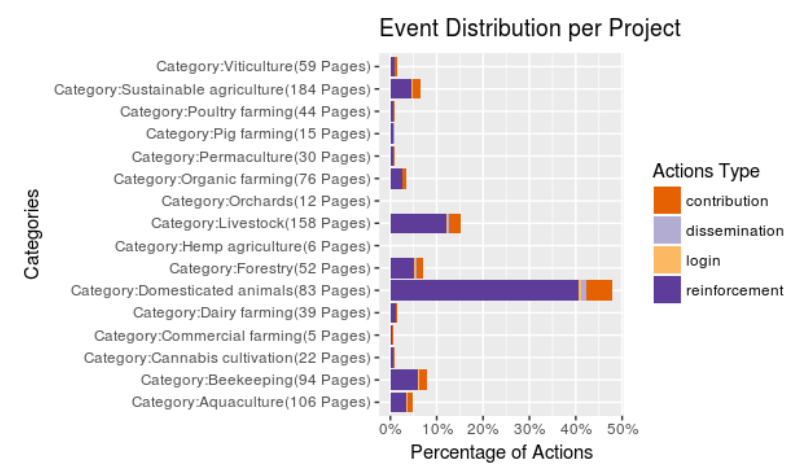

Figure 4: Events distribution per project.

a similar proportion of actions. The most representative actions are the reinforcement, then in fewer proportion contributions and finally dissemination and login. Surprisingly, the category with more received actions was "Domesticated animals" that only had 83 pages.

Regarding the evolution of the actions along the time (question Q4) we analyzed the accumulation of activities with a line chart. Figure 5 shows the accumulation of the Actions by type, regarding

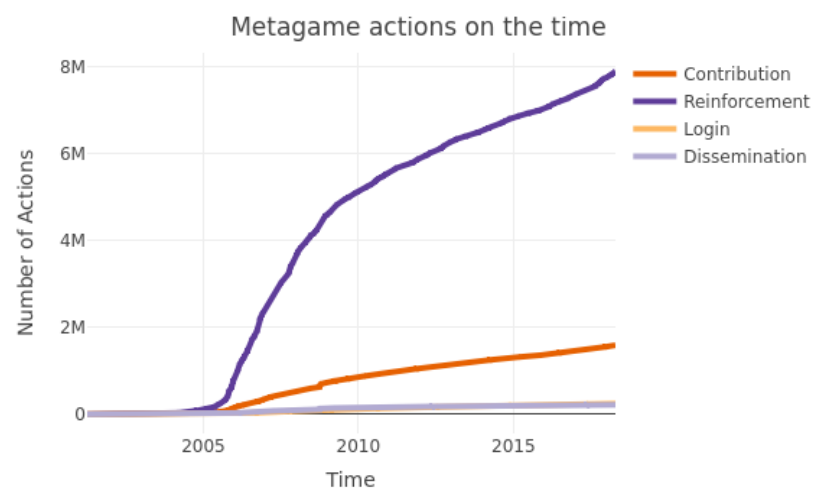

Figure 5: Metagame actions at the time.

Metagame, refers to the number of actions that registered until a determinate day. The axis "Number of Actions" represents the number of actions in millions, and the axis "Time" describes the moment each action was performed in Wikipedia. There is a line for each action type from 2001 to 2018. Regarding the beginning of the actions, the chart shows that in 2005 was initiated the most intense activities. Between 2005 and 2010 was a fast growth in the number of reinforcement. Also, from 2010 to our last event in April of 2018, the number of actions continued growing but more slowly. About each kind of action, the "Reinforcement" actions grew faster and more than the others. "Contribution" actions showed a slow growth. On the other hand, the "Dissemination" actions never had a relevant growth and, the "Login" actions cannot be seen. The big difference in the amount of reinforcement actions in comparison with the contribution actions represents that most of the activities that we have mapped as reinforcement could be actually contributions in Wikipedia. The construction of agriculture projects in Wikipedia is done in very small portions, and some of them can be confused as reinforcements instead of contributions.

About Metagame results, the Figure 6 shows for each project the distribution of the players per ranking. In the axis "Categories" there are the name of each category with the number of articles that It contains, in the axis "Percentage of Ranks" there are for each category a bar that represents the percentage of Users that participate in this category differentiated per their rank by color. Because the rank belongs to the player and the player could participate in many projects, It is interesting saw how the projects with fewer articles like the category "Hemp agriculture" or "Aquaculture" were carried out per players of the highest rank obtained in this simulation. It was related to that the less interesting or relevant articles were supported by, the more committed users. Also, It was possible to see that the majority of the users that interacted in some project were occasional users and because that they had the rank "Visitor". This Figure and the next helped to answered Q5.

The Figure 7 shows the total distribution of players in their ranks. The ranks obtained during the simulation were "Visitor", "Explorer" and "Citizen Scientist". Also, some ranks never were reached like "Prolific Citizen Scientist", "Committed Citizen Scientist". It showed that the difficulty was too high because in the 17 years that were 


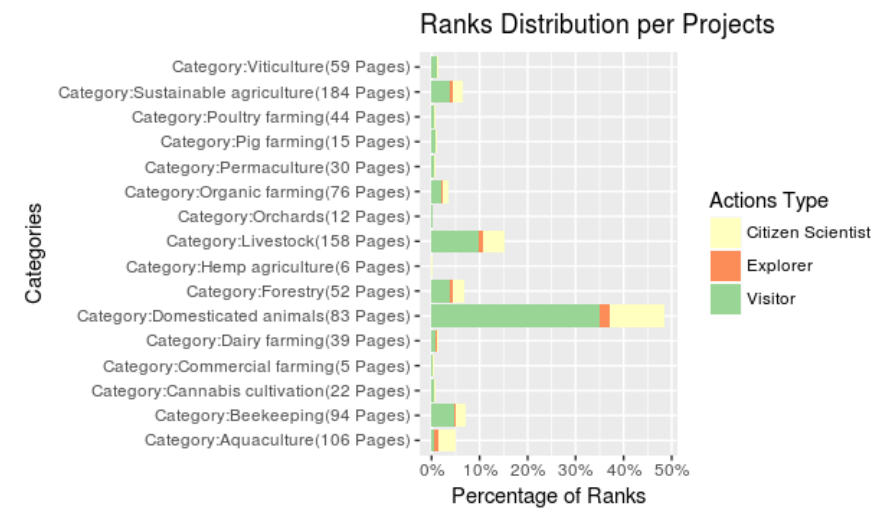

Figure 6: Metagame players per project by rank.

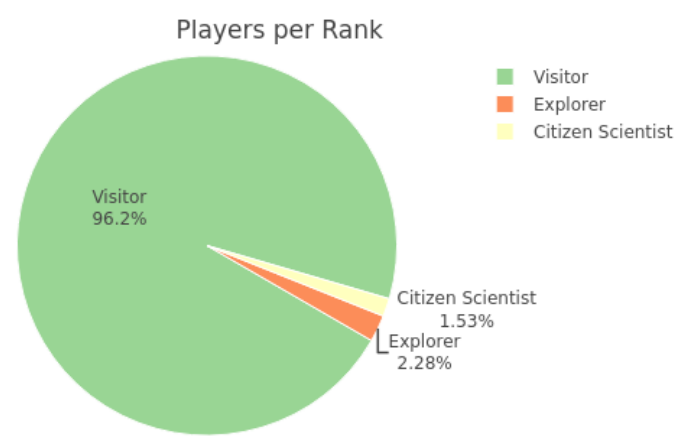

Figure 7: Metagame percentage of players per ranks.

covered by this simulation anybody could obtain a higher rank than "Citizen Scientist". This difficulty could be related to the way in that some of the actions were considered. For example, some actions of "Reinforcement" could be considered as "Contribution" because in Wikipedia some contributions are smaller than what was considered, like new sentences instead of paragraphs. Also, the distribution of the dataset in many projects could be too small, maybe if some of those projects were fused the player could gather more badges and access to higher ranks.

Regarding the delivery of badges at the time (question Q6) we analyzed the accumulation of badges with a line chart. Figure 8 shows the accumulation of badges delivered at each day per type of badge. The axis "Number of Badges" represents the number of badges in thousands, and the axis "Time" describes the moment each badge was earned by a player in Metagame. There is a line for each badge type from 2001 to 2018. A line near to the bottom meaning that the badges of this type of actions were obtained fewer times. Also, the lines that were nearest between refers that those lines were obtained a similar number of times. Also, a badge that it was no gather many times could be considered more difficult to obtain. An example of that was the case of the Dissemination actions. About nearest badges line, they could be considered balanced. An example

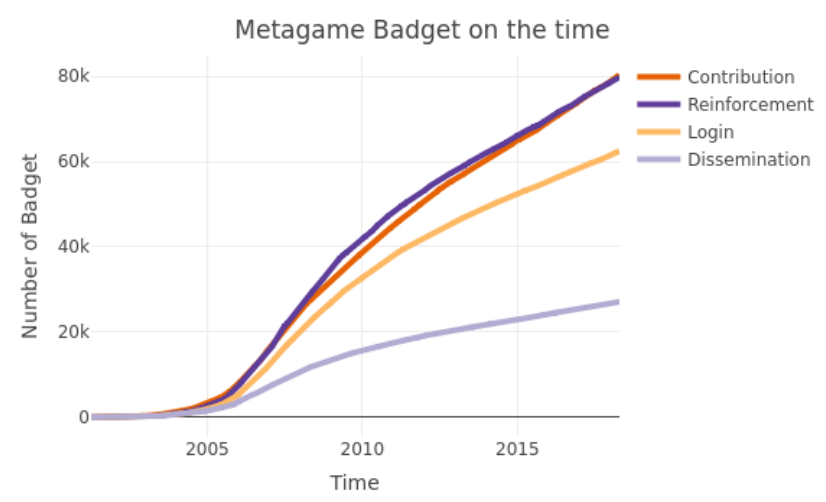

Figure 8: Delivered Metagame Badges at the time.

of that was the contribution, and the reinforcement delivered badges because even though there were more actions of reinforcement as was mention above, the requirements for the reinforcement badges was 5 times larger than for the contribution badges. Finally, another thing to consider at this step was if the dissemination activities have to be made easier, for example, adding more type of actions of dissemination or if It is better award more the actual actions. In an implementation on the production face of the wiki, this case is not tribal because this difficulty makes almost impossible access to the highest ranks and that could frustrate players.

\section{RELATED WORKS}

The work of Marques[14] had as objective obtain a system of knowledge management and construction, and mentions that it will be feed by experts and that It will be implemented using a Wiki environment. Also, to improve the participation of the users in the wiki gamification elements were included. Those gamification elements were the pluggings for Wikipedia called Xquiz ${ }^{7}$, to create a quiz and publish their results, and Activity Ranking that use a table of actions that defines a value with points, with what after a ranking of the users and their points. This approach is similar to the presented in this paper, but in the case of Metagame allow a more complex development of the gamification and progression mechanics. Related with the gamification of Wikipedia, the work of Narayan et. al.[12] presents an interactive tutorial, called The Wikipedia Adventure(TWA) that offers a structured and gamified introduction to Wikipedia. Their objective was to integrate new users into a Wikipedia community with Its complex norms. This strategy of gamification applied in a tutorial was expected to improve the integration of the new user while the attachment to Metagame had as objective improve and incentive the collaboration of all kind of users. Also, TWA was directly applied to the real Wikipedia projects, and in our case was developed as simulation over historical data. Finally, other work related to gamification in the knowledge building process but no implemented over wikis is developed by Senabre[1] and called Gamification Quest : * or GQ*. It focuses on presenting a mechanics and a game system that uses

${ }^{7}$ http://extensions.xwiki.org/xwiki/bin/view/Extension/XQuiz/AccessedonApril25th, 2018 
the student's notes and deliveries, collected by the GQ application. In a way to motivate him to improve his academic performance to progress in the game.

\section{CONCLUSION AND FURTHER WORK}

This work introduced a study of applying a gamification strategy in an agricultural collaborative knowledge building process. As the use of a gamification strategy is risky to apply in production, we adapted the Orta \& Ruiz conceptual framework [13] to run a simulation based on the historical data existing in Wikipedia using the Metagame pluggable gamification service.

The simulation included the development of tasks to extract relevant pages from Wikipedia, convert Wikipedia actions into Metagame actions, tune badges rules and run the simulation.

An evaluation was done over activity over 17 years. Results have shown that most of the edition actions were performed by a small group of active players. The type of activities are similarly distributed in the different projects, that means a similar amount of contributions, reinforcements, disseminations, and login. Surprisingly, Domesticated animals project with 83 pages has near of $50 \%$ of all the actions. Also, more of the actions in time are those considered as Reinforcement. The construction of agriculture projects in Wikipedia is done in very small portions and some of them can be confused as reinforcements instead of contributions. Finally, the level of difficulty of acquisition of badges was balanced for reinforcement and contribution, but it was too difficult for dissemination badges. This problem combined with the fact of have to many small projects stopped the evolution of players in the Metagame levels of expertise (Player rank). Even if It could be resolved when the editors realize of how to increment their rank, It could disagree with the objectives of the knowledge building process of Wikipedia. In another hand, a solution could be an improvement in the fine-tuning of the gamification.

This initial analysis opens several lines of further work. As the primary activity, we would like to configure new rules of mapping to analyze a better evolution in players ranks and badge collection. Understand the level of participation of a player and how much time it participates are other further lines. Because in this work only were obtained atemporal ranks and made cumulative analysis, if ranks based in time are obtained, It could be possible analyses the temporal behavior and how long takes at newer editors reach older editors. Finally, a more complete analysis of the whole Agriculture category and its pages will be proposed.

\section{Acknowledgments}

Authors of this publication acknowledge the contribution of the Project 691249, RUC-APS: Enhancing and implementing Knowledge based ICT solutions within high Risk and Uncertain Conditions for Agriculture Production Systems (www.ruc-aps.eu), funded by the European Union under their funding scheme H2020-MSCA-RISE2015.

\section{REFERENCES}

[1] Luis Alisandra Senabre. 2017. Gamification Quest:* Design and Development of a gamification game. (2017).

[2] Eskender Beza, Jonathan Steinke, Jacob van Etten, Pytrik Reidsma, Carlo Fadda, Sarika Mittra, Prem Mathur, and Lammert Kooistra. 2017. What are the prospects for citizen science in agriculture? \{Evidence\} from three continents on motivation and mobile telephone use of resource-poor farmers. PloS One 12, 5 (2017), e0175700. https://doi.org/10.1371/journal.pone. 0175700

[3] Brian Burke. 2012. Gamification 2020: What is the future of gamification. Gartner, Inc., Nov 5 (2012)

[4] Sebastian Deterding, Dan Dixon, Rilla Khaled, and Lennart Nacke. 2011. From \{Game\} \{Design\} \{Elements\} to \{Gamefulness\}: $\{$ Defining\} "\{Gamification\}". In Proceedings of the 15th \{International\} \{Academic\} \{MindTrek\}\{Conference\}: $\{$ Envisioning\} \{Future\} \{Media\} \{Environments\} (\{MindTrek\} '11). ACM, New York, NY, USA, 9-15. https://doi.org/10.1145/2181037.2181040

[5] Hannes Dohrn and Dirk Riehle. 2011. Design and implementation of the sweble wikitext parser: unlocking the structured data of wikipedia. In Proceedings of the 7th International Symposium on Wikis and Open Collaboration. 72-81.

[6] AdriÃąn Domínguez, Joseba Saenz-De-Navarrete, Luis De-Marcos, Luis Fernández-Sanz, Carmen Pagés, and JosÃl Javier Martínez-Herráiz. 2013. Gamifying learning experiences: Practical implications and outcomes. Computers and Education 63 (2013), 380-392. https://doi.org/10.1016/j.compedu.2012.12.020

[7] Oliver Ferschke, Torsten Zesch, and Iryna Gurevych. 2011. Wikipedia revision toolkit: efficiently accessing Wikipedia's edit history. In Proceedings of the 49th Annual Meeting of the Association for Computational Linguistics: Human Language Technologies: Systems Demonstrations. 97-102.

[8] Marc I Kellner, Raymond J Madachy, and David M Raffo. 1999. Software process simulation modeling: why? what? how? Journal of Systems and Software 46, 2-3 (1999), 91-105.

[9] Markus Krötzsch, Denny Vrandečić, and Max Völkel. 2006. Semantic \{MediaWiki\}. In The \{Semantic\} $\{W e b\}-\{I S W C\}$ 2006, Isabel Cruz, Stefan Decker, Dean Allemang, Chris Preist, Daniel Schwabe, Peter Mika, Mike Uschold, and Lora M Aroyo (Eds.). Springer Berlin Heidelberg, Berlin, Heidelberg, 935-942.

[10] Janette Lehmann, Mounia Lalmas, Elad Yom-Tov, and Georges Dupret. 2012. Models of user engagement. In International Conference on User Modeling, Adaptation, and Personalization. Springer, 164-175.

[11] Bo Leuf and Ward Cunningham. 2001. The Wiki Way: Quick Collaboration on the Web. Addison-Wesley Professional.

[12] Sneha Narayan, Jake Orlowitz, Jonathan Morgan, Benjamin Mako Hill, and Aaron Shaw. 2017. The Wikipedia Adventure: Field Evaluation of an Interactive Tutorial for New Users. In Proceedings of the 20th ACM Conference on Computer-Supported Cooperative Work \& Social Computing (CSCWâĂŹ17).

[13] Elena Orta and Mercedes Ruiz. 2016. A simulation and gamification approach for IT service management improvement. In International Conference on Software Process Improvement and Capability Determination. Springer, 84-97.

[14] Joaquim Pellicer Marquès. [n. d.]. Wiki y ludificación vinculados a un entorno de diagnóstico para la imágen. ([n. d.]).

[15] Santiago Pravisani. 2017. Metagame. In I Workshop de Ciencia Abierta y Ciudadana-Argentina (La Plata, 2017).

[16] Santiago Pravisani, Alejandro Fernandez, and Diego Torres. 2018. cientopolis/metagame-api \{V\}1.0.0. https://doi.org/10.5281/zenodo.1219096

[17] Diego Torres, Pascal Molli, Hala Skaf-Molli, and Alicia Diaz. 2012. Improving wikipedia with dbpedia. In Proceedings of the 21st International Conference on World Wide Web. ACM, 1107-1112.

[18] Diego Torres, Hala Skaf-Molli, Pascal Molli, and Alicia Díaz. 2013. Discovering wikipedia conventions using DBpedia properties. In International Workshop on Semantic Web Collaborative Spaces. Springer International Publishing, 115-144.

[19] Xiaoli Zhao and M J Bishop. 2011. Understanding and supporting online communities of practice: lessons learned from \{Wikipedia\}. Educational Technology Research and Development 59, 5 (2011), 711-735. https://doi.org/10.1007/ s11423-011-9204-7 